\title{
Comparison of four markers of hepatic fibrosis and hepatic function indices in patients with liver cirrhosis and hepatoma
}

\author{
Hong-Yu Ma ${ }^{1 \#}$, Lei Dong ${ }^{1 \#}$, Shou-Zhen Quan ${ }^{1}$, Ruo-Yi Li ${ }^{1}$, Xiang-Rong Wang ${ }^{2}$ \\ ${ }^{1}$ Clinical Laboratory Department, Air Force Medical Center, PLA, Beijing, China; ${ }^{2}$ Medical Assurance Center, Air Force Medical Center, PLA, \\ Beijing, China \\ Contributions: (I) Conception and design: HY Ma; (II) Administrative support: L Dong; (III) Provision of study materials or patients: SZ Quan; (IV) \\ Collection and assembly of data: RY Li; (V) Data analysis and interpretation: XR Wang; (VI) Manuscript writing: All authors; (VII) Final approval of \\ manuscript: All authors. \\ "These authors contributed equally to this work. \\ Correspondence to: Xiang-Rong Wang. Medical Assurance Center, Air Force Medical Center, PLA, No. 30 of Fucheng Street, Haidian District, Beijing \\ 100142, China. Email: wangxiangrongzz@163.com.
}

Background The present study aimed to compare four hepatic fibrosis markers [i.e., hyaluronic acid (HA), laminin (LN), procollagen III N-terminal peptide (PIIINP), and collagen type IV (CIV)] and 16 hepatic function indices in patients with liver cirrhosis of varying etiology.

Methods: The hepatic function indices and hepatic fibrosis markers were measured in 108 patients with liver cirrhosis and hepatoma using an automatic biochemical analyzer and luminescent immune analyzer. Twenty healthy controls were enrolled to compare the differences between liver cirrhosis and hepatoma of varying etiology and to analyze the correlations between the hepatic function indices and fibrosis markers.

Results: There was no correlation between alanine aminotransferase (ALT), total protein (TP), alkaline phosphatase (ALP), or the four markers of hepatic fibrosis in liver cirrhosis caused by hepatitis $\mathrm{B}(\mathrm{P}>0.05)$. Aspartate aminotransferase (AST) was positively correlated with HA ( $\mathrm{r}=0.428, \mathrm{P}=0.007), \mathrm{LN}(\mathrm{r}=0.458$, $\mathrm{P}=0.004)$, and $\mathrm{CIV}(\mathrm{r}=0.374, \mathrm{P}=0.021)$. Total bilirubin (TBIL) and direct bilirubin (DBIL) were positively correlated with LN (TBIL: $r=0.480, \mathrm{P}=0.002$; DBIL: $\mathrm{r}=0.457, \mathrm{P}=0.004$ ), PIIINP (TBIL: $\mathrm{r}=0.380, \mathrm{P}=0.017$; DBIL: $r=0.406, \mathrm{P}=0.011$ ), and CIV (TBIL: $\mathrm{r}=0.415, \mathrm{P}=0.010$; DBIL: $\mathrm{r}=0.400, \mathrm{P}=0.013$ ). Total bile acid (TBA) and $\gamma$-glutamyltranspeptidase (GGT) were positively correlated with PIIINP (TBA: r=0.363, P=0.025; GGT: $\mathrm{r}=0.353, \mathrm{P}=0.029$ ) and CIV (TBA: $\mathrm{r}=0.419, \mathrm{P}=0.009$; GGT: $\mathrm{r}=0.335, \mathrm{P}=0.040)$. Leucine aminopeptidase (LAP) was positively correlated with $\mathrm{LN}(\mathrm{r}=0.482, \mathrm{P}=0.002)$. Cholinesterase (CHE) (HA: $\mathrm{r}=-0.452, \mathrm{P}=0.004, \mathrm{LN}$ : $r=-0.336, P=0.039$; PIIINP: $r=-0.468, P=0.003$; CIV: $r=-0.485, P=0.002)$, prealbumin (PA) (HA: $r=-0.575$, $\mathrm{P}=0.000, \mathrm{LN}: \mathrm{r}=-0.413, \mathrm{P}=0.010$; PIIINP: $\mathrm{r}=-0.344, \mathrm{P}=0.035$; CIV: $\mathrm{r}=-0.371, \mathrm{P}=0.022$ ), albumin (ALB) (HA: $r=-0.541, \mathrm{P}=0.000$, LN: $r=-0.373, \mathrm{P}=0.021$; PIIINP: $\mathrm{r}=-0.353, \mathrm{P}=0.030$; $\mathrm{CIV}: \mathrm{r}=-0.415, \mathrm{P}=0.010$ ), and superoxide dismutase (SOD) (HA: $r=-0.334, \mathrm{P}=0.040, \mathrm{LN}: \mathrm{r}=-0.347, \mathrm{P}=0.033$; PIIINP: $\mathrm{r}=-0.487, \mathrm{P}=0.002$; CIV: $\mathrm{r}=-0.536, \mathrm{P}=0.001)$ were negatively correlated with the four markers of hepatic fibrosis. There was no correlation between ALT, AST, TBIL, TP, ALP, GGT, or the four hepatic fibrosis markers in hepatoma caused by hepatitis $\mathrm{B}(\mathrm{P}>0.05)$. Meanwhile, DBIL and TBA were positively correlated with CIV (DBIL: $\mathrm{r}=0.519, \mathrm{P}=0.023$; TBA: $\mathrm{r}=0.563, \mathrm{P}=0.012)$, while CHE ( $\mathrm{r}=-0.604, \mathrm{P}=0.006)$, ALB ( $\mathrm{r}=-0.564, \mathrm{P}=0.012)$, and SOD ( $\mathrm{r}=-0.489, \mathrm{P}=0.034)$ were negatively correlated with $\mathrm{CIV}$. Moreover, PA was negatively correlated with $\mathrm{LN}(\mathrm{r}=-0.510, \mathrm{P}=0.026)$ and $\mathrm{CIV}(\mathrm{r}=-0.696, \mathrm{P}=0.001)$.

Conclusions: The concentrations of the serological indices differed significantly based on the specific liver cirrhosis etiology. There was a strong correlation between the hepatic function indices and four hepatic fibrosis markers. Thus, the detection of these markers might improve the diagnosis and treatment of hepatoma.

Keywords: Fibrosis-4 index; hyaluronic acid; laminin; procollagen III N-terminal peptide; collagen type IV; cirrhosis; hepatic cancer 
Submitted Aug 17, 2020. Accepted for publication Feb 04, 2021.

doi: 10.21037/apm-20-1623

View this article at: http://dx.doi.org/10.21037/apm-20-1623

\section{Introduction}

Fibrosis is an intermediate stage in the progression of chronic hepatitis to liver cirrhosis (1). At present, pathological biopsies, imaging examinations, and serological examinations are used to clinically examine hepatic lesions (2). The pathological analyses are mainly based on liver biopsy, which is regarded as the gold standard; however, there are many disadvantages to this approach, such as errors caused by blind puncture or uneven hepatic lesions. Moreover, a liver puncture is an invasive examination that cannot be observed dynamically; thus, it may not be accepted by most patients (3).

Imaging examinations mainly consist of B-ultrasound and computed tomography, but pathological imaging characteristics can only generally be identified when there are false lobules or hepatic occupation. Thus, imaging cannot provide an early diagnosis. In recent years, transient elastography has shown good application prospects, but it requires further verification (4).

Serological diagnosis is the most widely used noninvasive diagnostic method, as it allows for convenient sample collection and can be observed dynamically; however, its specificity is relatively low (5). As a biomarker of fibrosis, the $\mathrm{N}$-terminal peptide of type III procollagen (PIIINP) has reached a limited clinical application with 76-78\% sensitivity and $71-81 \%$ specificity, which can be increased to $88 \%$ if combined with additional collagen fragment markers (6). However, PIIINP is not a liverspecific biomarker and has not gained widespread acceptance. Hyaluronic acid (HA) may relatively be the best biomarker of fibrosis, with high sensitivity and specificity (7). Due to its higher negative predictive value compared with a positive predictive value, HA is mainly used to exclude advanced fibrosis and cirrhosis and is considered a valid fibrosis biomarker. Laminin (LN) could be a predictor of portal hypertension (8). The traditional biomarkers of hepatic function include alanine aminotransferase (ALT) and aspartate aminotransferase (AST). However, few parameters can be regarded as directly related to fibrogenesis.

Therefore, the present study aimed to compare hepatic function indices and four markers of hepatic fibrosis in patients with liver cirrhosis of varying etiology. We further analyzed the correlations among the hepatic fibrosis markers, hepatic function indices, and other clinical indices. These findings may improve clinical diagnosis of and timely treatment for liver cirrhosis and hepatoma. We present the following article in accordance with the MDAR checklist (available at http://dx.doi.org/10.21037/apm-20-1623).

\section{Methods}

\section{Patients}

From January to May 2017, 108 inpatients from the Air Force General Hospital of the People's Liberation Army were enrolled (male, $n=77$; female, $n=31$ ), with an age range of 24-87 years, an average age of 53 years, and a disease duration of 1 month-40 years. Within the same period, 20 healthy subjects from our hospital were enrolled in the control group to undergo physical examination (male, $\mathrm{n}=7$; female, $\mathrm{n}=13$ ). All subjects in the control group had normal hepatic function, no history of viral hepatitis or alcoholic hepatic disease, no history of alcohol abuse, and no fatty liver when imaged using ultrasound. Serious primary diseases (including cardiovascular, cerebrovascular, pulmonary, renal, endocrine, and hematopoietic diseases), and incomplete data were excluded. There were no significant differences in age or sex between the groups $(\mathrm{P}>0.05)$, and the data were comparable. The study was conducted in accordance with the Declaration of Helsinki (as revised in 2013). The study was approved by ethics board of Air Force Medical Center, PLA (approval number: 20161201) and informed consent was taken from all the patients.

\section{Liver cirrhosis caused by hepatitis B}

A total of 38 patients with liver cirrhosis caused by hepatitis $B$ were enrolled (male, $n=32$; female, $n=6$ ), with an age range of 31-81 years, an average age of 50 years, and a disease duration of $1-40$ years. The diagnostic criteria were as follows: (I) hepatic dysfunction; (II) clinical manifestation of portal vein hypertension; (III) liver cirrhosis detected by imaging; (IV) hepatitis B surface antigen ( $\mathrm{HBsAg}$ ) positivity. Patients with other types of liver cirrhosis. 


\section{Liver cirrhosis caused by hepatitis $\mathbf{C}$}

Two male patients with liver cirrhosis caused by hepatitis $\mathrm{C}$ were enrolled, with an age range of 58-70 years, an average age of 64 years, and a disease duration of 2-9 years. The diagnostic criteria were as follows: (I) hepatic dysfunction; (II) clinical manifestation of portal vein hypertension; (III) liver cirrhosis detected by imaging; (IV) serum anti-HCV positivity. Patients with other types of liver cirrhosis.

\section{Alcoholic liver cirrhosis}

Seven patients with alcoholic liver cirrhosis were enrolled (male, $n=6$; female, $n=1$ ), with an age range of 44-74 years, an average age of 62 years, and a disease duration of 1 month-7 years. The diagnostic criteria were as follows: (I) hepatic dysfunction; (II) clinical manifestation of portal vein hypertension; (III) liver cirrhosis detected by imaging; (IV) a long history of alcohol intake. Patients with other types of liver cirrhosis.

\section{Congestive liver cirrhosis}

Nine patients with congestive liver cirrhosis were enrolled (male, $n=5$; female, $n=4$ ), with an age range of 24-50 years, an average age of 42 years, and a disease duration of 1 month-10 years. The diagnostic criteria were as follows: (I) hepatic dysfunction; (II) clinical manifestation of portal vein hypertension; (III) liver cirrhosis detected by imaging; (IV) obstruction in the hepatic vein and/or inferior vena cava. Patients with other types of liver cirrhosis.

\section{Autoimmune liver cirrhosis}

Five female patients with autoimmune liver cirrhosis were enrolled, with an age range of 49-81 years, an average age of 62 years, and a disease duration of 3-30 years. The diagnostic criteria were as follows: (I) hepatic dysfunction; (II) clinical manifestation of portal vein hypertension; (III) liver cirrhosis detected by imaging; (IV) antimitochondrial antibody positivity. Patients with other types of liver cirrhosis.

\section{Occult liver cirrhosis}

A total of 18 patients with occult liver cirrhosis were enrolled (male, $\mathrm{n}=8$; female, $\mathrm{n}=10$ ), with an age range of 28-72 years, an average age of 51 years, and a disease duration of 1 month-23 years. The diagnostic criteria were as follows: (I) hepatic dysfunction; (II) clinical manifestation of portal vein hypertension; (III) liver cirrhosis detected by imaging; (IV) liver cirrhosis that could not be explained by other known etiologies. Patients with other types of liver cirrhosis.

\section{Hepatoma caused by hepatitis B}

A total of 19 patients with liver cirrhosis caused by hepatitis B were enrolled (male, $n=17$; female, $n=2$ ), with an age range of 39-78 years, an average age of 54 years, and a disease duration of 3 months -30 years. The diagnostic criteria were as follows: (I) two types of imaging examinations indicated a characteristic hepatoma with a mass $>2 \mathrm{~cm}$, one type of imaging examination indicated a characteristic hepatoma with a mass $>2 \mathrm{~cm}$ with an alphafetoprotein (AFP) concentration of $\geq 400 \mu \mathrm{g} / \mathrm{L}$, or hepatoma confirmed by hepatic histology; (II) HBsAg positivity. Patients with other types of liver cancer.

\section{Hepatoma caused by hepatitis $\mathbf{C}$}

Three patients with liver cirrhosis caused by hepatitis C were enrolled (male, $n=1$; female, $n=2$ ), with an age range of 60-87 years, an average age of 71 years, and a disease duration of 3-25 years. The diagnostic criteria were as follows: (I) two types of imaging examinations indicated a characteristic hepatoma with a mass $>2 \mathrm{~cm}$, one kind of imaging examination indicated a characteristic hepatoma with a mass $>2 \mathrm{~cm}$ together with an AFP concentration of $\geq 400 \mu \mathrm{g} / \mathrm{L}$, or hepatoma confirmed by hepatic histology; (II) $\mathrm{HBcAg}$ positivity. Patients with other types of liver cancer.

\section{Primary hepatoma}

Six patients with primary hepatoma were enrolled (male, $\mathrm{n}=5$; female $\mathrm{n}=1$ ), with an age range of 49-86 years, an average age of 65 years, and a disease duration of 1 month-28 years. The diagnostic criteria were as follows: two types of imaging examination indicated a characteristic hepatoma with a mass $>2 \mathrm{~cm}$, one kind of imaging examination indicated a characteristic hepatoma with a mass $>2 \mathrm{~cm}$ together with an AFP concentration of $\geq 400 \mu \mathrm{g} / \mathrm{L}$, or hepatoma confirmed by hepatic histology.

\section{Data collection}

\section{Sample collection}

Before treatment initiation, fasting venous blood was collected from all patients the morning after hospitalization. The samples were put into EDTA-K2 anticoagulant tubes and centrifuged at $3,500 \mathrm{rpm} / \mathrm{min}$ for 10 minutes; the separated serum was frozen at $-80^{\circ} \mathrm{C}$ for further analysis. 


\section{Detection of hepatic function}

The changes in hepatic function in each group were measured using a Hitachi 7600 automatic biochemical analyzer (Hitachi, Tokyo, Japan) and corresponding reagents. In total, 16 hepatic function indices were measured: ALT, AST, total bilirubin (TBIL), direct bilirubin (DBIL), total protein (TP), albumin (ALB), the ratio of ALB to globulin $(\mathrm{A} / \mathrm{G})$, prealbumin $(\mathrm{PA})$, alkaline phosphatase (ALP), $\gamma$-glutamyltranspeptidase (GGT), total bile acid (TBA), cholinesterase (CHE), serum amylase (AMY), $\alpha$-L-fucosidase (AFU), leucine aminopeptidase (LAP), and superoxide dismutase (SOD).

\section{Detection of the four markers of hepatic fibrosis}

Using HA as an example, double-antibody sandwich immunochromatography was adopted, and an upconversion immunoanalyzer was used. The reaction area (T-line) of the nitrocellulose membrane on the test card was coated with the serum hepatic fibrosis index HA antibody (cat no. 20160915, KeyGen Gene Technology Co., Ltd., Beijing China), and the quality control area (C-line) was coated with sheep anti-mouse antibody (cat no. 20160318, KeyGen Gene Technology Co., Ltd.). The scientific principle behind this approach is that particles emit light under excitation, and the ratio of the T-line signal to the $\mathrm{C}$-line signal (T/C) is directly proportional to the concentration of antigen in the sample. The test card is inserted into the matching up-conversion luminescent immunoanalyzer, and the concentration of indicator in the sample is read directly from the instrument screen. Serum HA was measured using this method. All reagents and instruments used in the present study were sourced from Beijing Rejing Biotechnology Co., Ltd.

\section{Statistical analysis}

The SPSS 22.0 software was used to perform the statistical analysis. The data were expressed as the mean \pm standard deviation from at least three independent measurements, each performed in triplicate. The single-sample Kolmogorov-Smirnov test was used to examine whether the sample data were normally distributed. A variance analysis and the Bonferroni correction were used for multiple group comparisons. Countable data were expressed by rate (\%). The $\chi^{2}$ test was used for group comparisons, while Pearson's linear correlation analysis was used for the variable correlation analysis. A $\mathrm{P}$ value $<0.05$ was considered statistically significant.

\section{Results}

\section{Comparison of the general characteristics between groups}

There were no significant differences in the general characteristics, e.g., height, weight, and body mass index, between the groups $(\mathrm{P}>0.05$; Table 1$)$. The hepatic function indices and the four hepatic fibrosis markers were comparable.

\section{Comparison of hepatic function indices between groups}

An analysis of variance was used to compare the means of multiple groups. The differences in the serological hepatic function indices between groups were statistically significant $(\mathrm{P}<0.05)$. Group comparisons were carried out using the Bonferroni correction, and the results are shown in Tables 2-5.

Comparison of serum ALT between groups: the ALT concentration was lowest in the control group and highest in the group with hepatoma caused by hepatitis B. A pairwise comparison revealed significant differences in the ALT concentration between the group with liver cirrhosis caused by hepatitis $\mathrm{B}$, the group with hepatoma caused by hepatitis $\mathrm{B}$, and the control group $(\mathrm{P}<0.05)$.

Comparison of serum AST between groups: The AST concentration was lowest in the control group and highest in the group with hepatoma caused by hepatitis B. A pairwise comparison showed significant differences in the AST concentration between the group with liver cirrhosis caused by hepatitis $B$, the control group, and the group with hepatoma caused by hepatitis $\mathrm{B}(\mathrm{P}<0.05)$.

Comparison of serum TBIL between groups: the TBIL concentration was lowest in the control group and highest in the autoimmune liver cirrhosis group. There were significant differences in the TBIL concentration in the group with liver cirrhosis caused by hepatitis $\mathrm{B}$, the group with congestive liver cirrhosis, and the group with autoimmune liver cirrhosis compared with the group with hepatoma caused by hepatitis B and the control group $(\mathrm{P}<0.05)$.

Comparison of serum DBIL between groups: The DBIL concentration was lowest in the control group and highest in the autoimmune liver cirrhosis group. There were significant differences in the DBIL concentration between the autoimmune liver cirrhosis group, the group with liver cirrhosis caused by hepatitis B, the group with hepatoma caused by hepatitis $\mathrm{B}$, the occult liver cirrhosis group, the group with hepatoma caused by hepatitis $\mathrm{C}$, the 
Table 1 Comparison of the general characteristics between groups $\left(\bar{x}_{ \pm \mathrm{S}}\right)$

\begin{tabular}{lccc}
\hline Indicators & Height $(\mathrm{m})$ & Weight $(\mathrm{kg})$ & $\mathrm{BMI}\left(\mathrm{kg} / \mathrm{m}^{2}\right)$ \\
\hline Control group & $1.68 \pm 0.09$ & $62.96 \pm 11.48$ & $22.31 \pm 2.81$ \\
Liver cirrhosis caused by hepatitis B & $1.70 \pm 0.06$ & $68.17 \pm 9.74$ & $23.59 \pm 3.05$ \\
Liver cirrhosis caused by hepatitis C & $1.76 \pm 0.06$ & $59.00 \pm 4.00$ & $19.29 \pm 2.51$ \\
Alcoholic liver cirrhosis & $1.70 \pm 0.02$ & $58.43 \pm 5.29$ & $20.10 \pm 1.57$ \\
Congestive liver cirrhosis & $1.64 \pm 0.05$ & $61.89 \pm 9.63$ & $22.95 \pm 3.38$ \\
Autoimmune liver cirrhosis & $1.63 \pm 0.03$ & $57.60 \pm 6.25$ & $21.67 \pm 1.60$ \\
Occult liver cirrhosis & $1.67 \pm 0.09$ & $62.69 \pm 14.19$ & $22.19 \pm 3.60$ \\
Hepatoma caused by hepatitis B & $1.70 \pm 0.05$ & $63.89 \pm 7.22$ & $22.19 \pm 2.63$ \\
Hepatoma caused by hepatitis C & $1.65 \pm 0.01$ & $65.33 \pm 6.94$ & $23.93 \pm 2.80$ \\
Primary hepatoma & $1.71 \pm 0.06$ & $73.17 \pm 8.01$ & $24.84 \pm 1.27$ \\
F values & 1.621 & 1.744 & $>0.05$ \\
P values & $>0.05$ & & $>0.05$ \\
\hline
\end{tabular}

Table 2 Comparison of hepatic function indices between groups $(\bar{x} \pm \mathrm{s})$

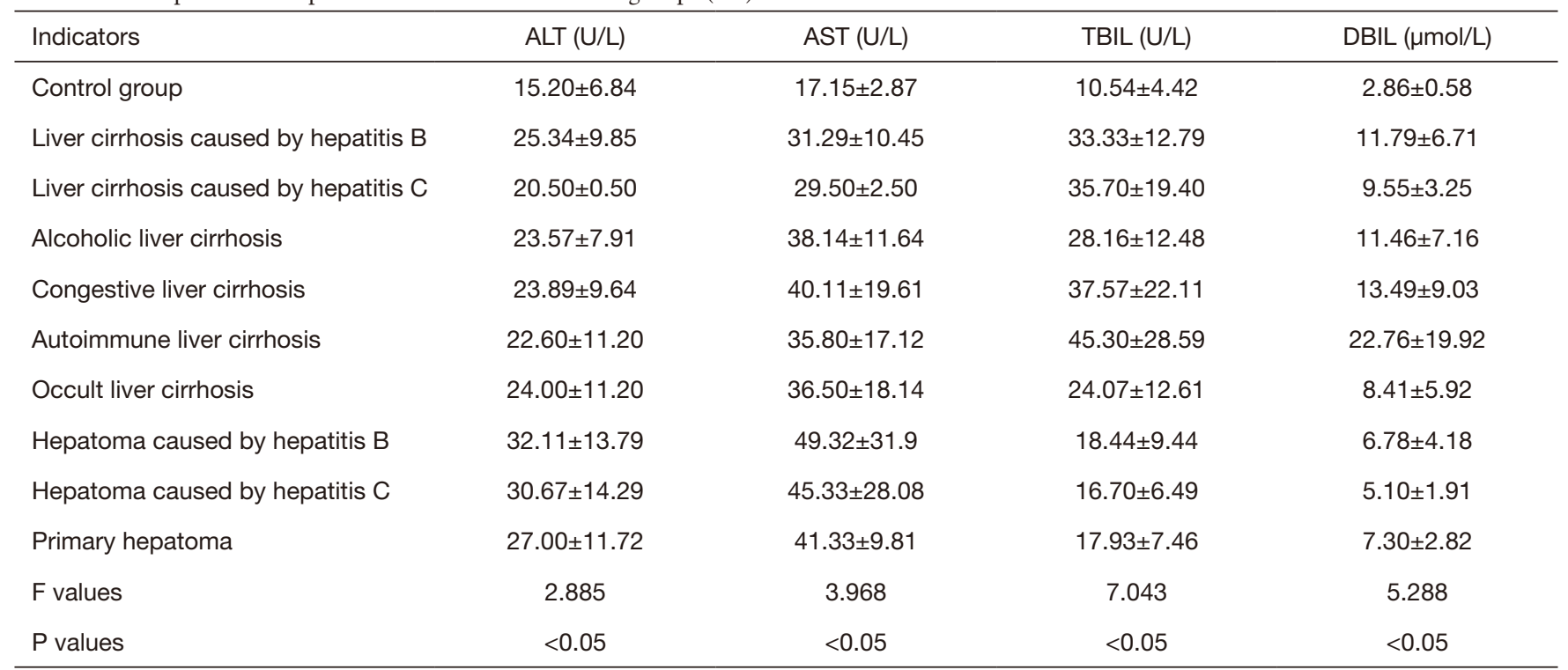

primary hepatoma group, and the control group $(\mathrm{P}<0.05)$. In addition, there were significant differences in the DBIL concentration between the group with liver cirrhosis caused by hepatitis $\mathrm{B}$, the congestive liver cirrhosis group, and the control group $(\mathrm{P}<0.05$; Table 2$)$.

Comparison of serum TP between groups: the TP concentration was lowest in the group with liver cirrhosis caused by hepatitis B and highest in the control group. A pairwise comparison revealed that there were significant differences in the TP concentration between the group with liver cirrhosis caused by hepatitis $\mathrm{B}$, the congestive liver cirrhosis group, the occult liver cirrhosis group, and the control group $(\mathrm{P}<0.05)$.

Comparison of serum ALB between groups: the ALB concentration was highest in the control group. A pairwise contrast revealed that there were significant differences in the ALB concentration between the control group and the other groups $(\mathrm{P}<0.05)$, except for the group with liver 
Table 3 Comparison of hepatic function indices between groups $\left(\bar{x}_{ \pm \mathrm{S}}\right)$

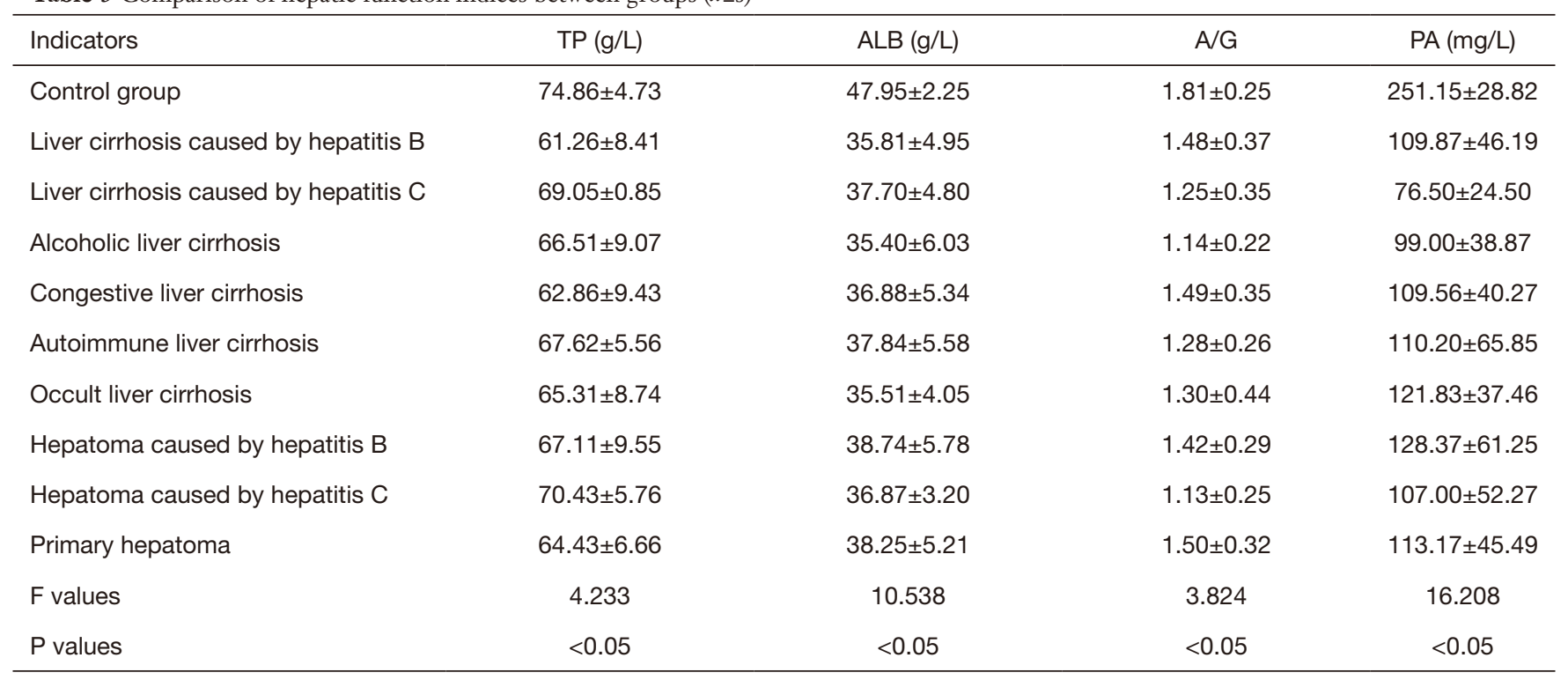

Table 4 Comparison of hepatic function indices between groups $(\bar{x} \pm \mathrm{s})$

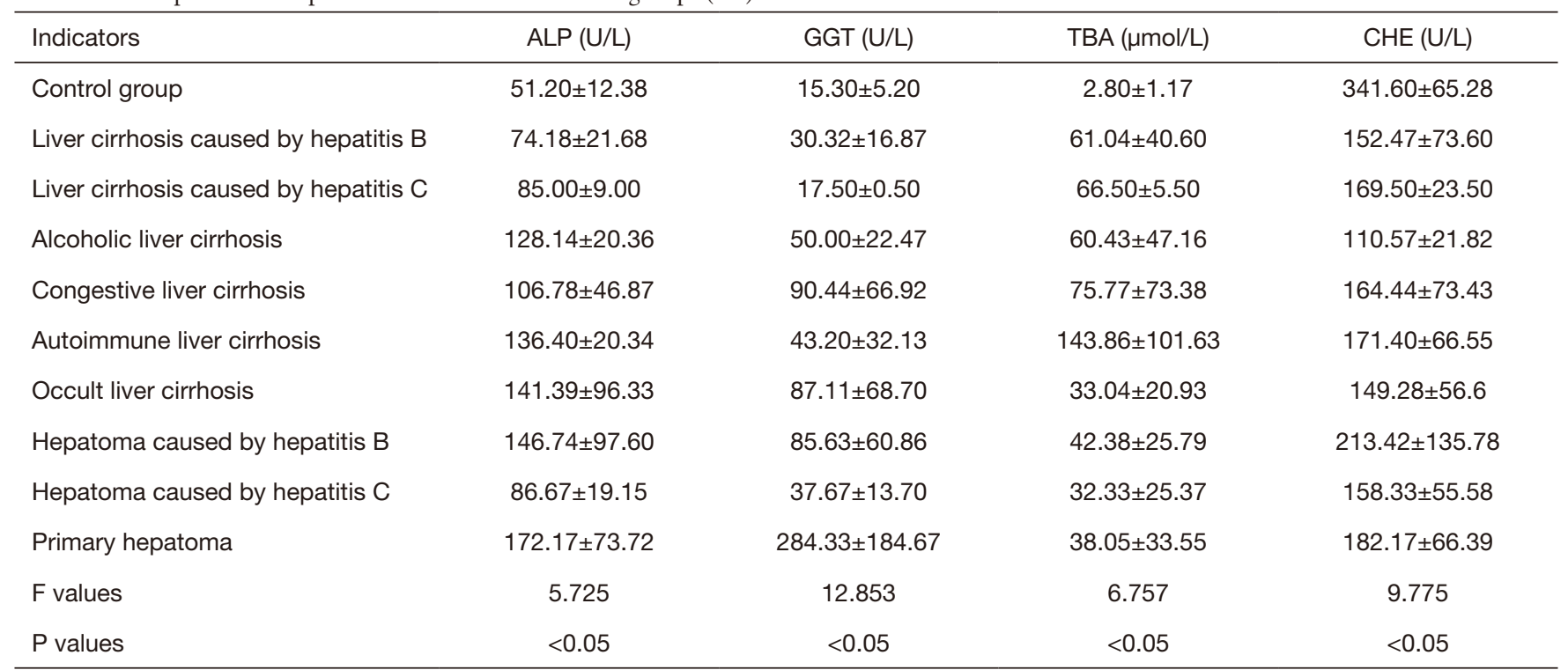

cirrhosis caused by hepatitis $\mathrm{C}$.

Comparison of $\mathrm{A} / \mathrm{G}$ between groups: the $\mathrm{A} / \mathrm{G}$ was highest in the control group. A pairwise comparison revealed significant differences in the $\mathrm{A} / \mathrm{G}$ between the group with liver cirrhosis caused by hepatitis $B$, the alcoholic liver cirrhosis group, the occult liver cirrhosis group, the group with hepatoma caused by hepatitis $\mathrm{B}$, and the control group $(\mathrm{P}<0.05)$.

Comparison of serum PA between groups: the PA concentration was highest in the control group and lowest in the group with liver cirrhosis caused by hepatitis C. A pairwise comparison revealed significant differences in the PA concentration between all nine groups compared with the control group $(\mathrm{P}<0.05)$. The PA concentration in the control group was significantly higher compared with that of the other groups (Table 3).

Comparison of serum ALP between groups: The ALP concentration was highest in the primary hepatoma group 
Table 5 Comparison of hepatic function indices between groups $\left(\bar{x}_{ \pm \mathrm{S}}\right)$

\begin{tabular}{|c|c|c|c|c|}
\hline Indicators & AMY (U/L) & AFU (U/L) & LAP (U/L) & $\mathrm{SOD}(\mathrm{U} / \mathrm{mL})$ \\
\hline Liver cirrhosis caused by hepatitis B & $58.92 \pm 21.42$ & $20.71 \pm 6.40$ & $61.24 \pm 19.91$ & $130.19 \pm 29.67$ \\
\hline Liver cirrhosis caused by hepatitis $C$ & $52.00 \pm 13.00$ & $11.00 \pm 6.00$ & $52.50 \pm 0.50$ & $131.50 \pm 8.50$ \\
\hline Alcoholic liver cirrhosis & $58.43 \pm 19.72$ & $26.57 \pm 8.53$ & $85.29 \pm 30.84$ & $124.00 \pm 22.86$ \\
\hline Autoimmune liver cirrhosis & $61.40 \pm 17.40$ & $21.40 \pm 7.36$ & $101.00 \pm 48.10$ & $132.88 \pm 30.69$ \\
\hline Occult liver cirrhosis & $47.89 \pm 17.89$ & $22.56 \pm 7.11$ & $85.94 \pm 39.63$ & $119.51 \pm 32.38$ \\
\hline Hepatoma caused by hepatitis B & $64.32 \pm 26.97$ & $29.00 \pm 6.73$ & $93.11 \pm 41.76$ & $125.56 \pm 29.10$ \\
\hline Hepatoma caused by hepatitis $C$ & $62.33 \pm 18.35$ & $21.00 \pm 7.35$ & $64.00 \pm 16.99$ & $115.47 \pm 6.85$ \\
\hline$P$ values & $>0.05$ & $<0.05$ & $<0.05$ & $<0.05$ \\
\hline
\end{tabular}

and lowest in the control group. A pairwise comparison revealed significant differences in the ALP concentration between the group with liver cirrhosis caused by hepatitis B and the occult liver cirrhosis group as well as the group with hepatoma caused by hepatitis B and the primary hepatoma group $(\mathrm{P}<0.05)$. Significant differences in the ALP concentration were also observed between the occult liver cirrhosis group, the group with hepatoma caused by hepatitis $\mathrm{B}$, the primary hepatoma group, and the control group $(\mathrm{P}<0.05)$.

Comparison of serum GGT between groups: the GGT concentration was relatively low in the control group and the group with liver cirrhosis caused by hepatitis C. A pairwise comparison revealed a significant difference in the GGT concentration between the primary hepatoma group and the other nine groups $(\mathrm{P}<0.05)$. The concentration in the primary hepatoma group was significantly higher compared with that in the other groups. In addition, there were significant differences in the GGT concentration between the occult liver cirrhosis group, the group with hepatoma caused by hepatitis B, and the control group $(\mathrm{P}<0.05)$.

Comparison of serum TBA between groups: the TBA concentration was highest in the autoimmune liver cirrhosis group and lowest in the control group. The results of the pairwise comparison revealed a significant difference in the TBA concentration between the autoimmune liver cirrhosis group and the other groups $(\mathrm{P}<0.05)$, except for the group with liver cirrhosis caused by hepatitis $\mathrm{C}$ and the congestive liver cirrhosis group. The TBA concentration in the autoimmune liver cirrhosis group was significantly higher compared with that in the other groups. Moreover, there were significant differences in the TBA concentration between the group with liver cirrhosis caused by hepatitis B, the congestive liver cirrhosis group, and the control group $(\mathrm{P}<0.05)$.

Comparison of serum CHE between groups: The CHE concentration in all groups was significantly lower compared with that in the control group. The results of the pairwise comparison revealed significant differences in the CHE concentration between the control group and all other groups, except for the group with liver cirrhosis caused by hepatitis $\mathrm{C}(\mathrm{P}<0.05$; Table 4$)$.

Comparison of serum AMY between groups: There were no significant differences in the AMY concentration between any of the groups $(\mathrm{P}>0.05)$.

Comparison of serum AFU between groups: The AFU concentration was lowest in the group with liver cirrhosis caused by hepatitis $\mathrm{C}$ and highest in the group with hepatoma caused by hepatitis B. A pairwise comparison revealed significant differences between the group with liver cirrhosis caused by hepatitis $\mathrm{B}$, the group with liver cirrhosis caused by hepatitis $\mathrm{C}$, the control group, and the group with hepatoma caused by hepatitis $\mathrm{B}(\mathrm{P}<0.05)$. There was also a significant difference in the concentration between the primary hepatoma group and the control group $(\mathrm{P}<0.05)$.

Comparison of serum LAP between groups: The LAP concentration was lowest in the control group and 
relatively high in the autoimmune liver cirrhosis group and the group with hepatoma caused by hepatitis B. The results of the pairwise comparison revealed a significant difference between the group with liver cirrhosis caused by hepatitis $\mathrm{B}$, the group with hepatoma caused by hepatitis $\mathrm{B}$, and the primary hepatoma group $(\mathrm{P}<0.05)$. There were also significant differences between the autoimmune liver cirrhosis group, the occult liver cirrhosis group, the group with hepatoma caused by hepatitis B, the primary hepatoma group, and the control group $(\mathrm{P}<0.05)$.

Comparison of serum SOD between groups: the SOD concentration was highest in the control group. A pairwise comparison revealed a significant difference in the SOD concentration between the occult liver cirrhosis group, the group with hepatoma caused by hepatitis $\mathrm{B}$, and the control group $(\mathrm{P}<0.05 ;$ Table 5$)$.

\section{Comparison of the four markers of hepatic fibrosis between groups}

Comparison of serum HA between groups: the HA concentration was lowest in the control group. A pairwise comparison revealed a significant difference in the HA concentration between the group with liver cirrhosis caused by hepatitis $\mathrm{B}$, the alcoholic liver cirrhosis group, the autoimmune liver cirrhosis group, the occult liver cirrhosis group, and the control group $(\mathrm{P}<0.05)$.

Comparison of serum LN between groups: The LN concentration was lowest in the control group. A pairwise comparison revealed a significant difference in the $\mathrm{LN}$ concentration between the group with liver cirrhosis caused by hepatitis B, the group with hepatoma caused by hepatitis $\mathrm{B}$, the primary hepatoma group, and the autoimmune liver cirrhosis group $(\mathrm{P}<0.05)$. Significant differences were also observed between the control group and the other groups, excluding the group with liver cirrhosis caused by hepatitis $\mathrm{C}$ and the three hepatoma groups $(\mathrm{P}<0.05)$.

Comparison of serum PIIINP between groups: The PIIINP concentration was lowest in the control group. The results of the pairwise comparison revealed a significant difference in the PIIINP between the group with liver cirrhosis caused by hepatitis $\mathrm{B}$, the occult liver cirrhosis group, and the alcoholic liver cirrhosis group $(\mathrm{P}<0.05)$. Significant differences also existed between the alcoholic liver cirrhosis group, the autoimmune liver cirrhosis group, the group with hepatoma caused by hepatitis $\mathrm{C}$, and the control group $(\mathrm{P}<0.05)$.

Comparison of serum CIV between groups: The serum
CIV concentration was lowest in the control group. A pairwise comparison revealed a significant difference in the concentration between the alcoholic liver cirrhosis group, the autoimmune liver cirrhosis group, and the control group $(\mathrm{P}<0.05$; Table 6).

The correlation analysis between the serum hepatic function indices and HA, LN, PIIINP, and CIV in the group with liver cirrhosis caused by hepatitis B revealed that ALT, TP, and ALP were not correlated with the four hepatic fibrosis markers in this group while AST was positively correlated with the four markers. Moreover, TBIL and DBIL were positively correlated with LN, PIIINP, and CIV, and TBA and GGT were positively correlated with PIIINP and CIV. Additionally, LAP was positively correlated with LN, while CHE, PA, ALB, and SOD were negatively correlated with the four hepatic fibrosis markers (Table 7).

The correlation analysis between the serum hepatic function indices and HA, LN, PNIIINP, and CIV in the group with hepatoma caused by hepatitis B revealed no correlation between ALT, AST, TBIL, TP, ALP, or GGT and the four markers. Moreover, DBIL and TBA were positively correlated with CIV, while CHE, ALB, and SOD were negatively correlated with CIV. Finally, PA was negatively correlated with LN and CIV (Table 8).

\section{Discussion}

Approximately 300 million people in China suffer from liver diseases, including viral hepatitis, non-alcoholic fatty hepatic disease, and alcoholic hepatic disease. Nearly 400,000 people die from liver cancer every year, accounting for $51 \%$ of the total number of liver cancer deaths worldwide. Since hepatic diseases increase the societal and medical burden in China (9), it is important to dynamically monitor changes in hepatic function, delay the progression of liver cirrhosis, reduce the incidence of liver cancer, and assist in clinical diagnosis and treatment through serological detection (10).

In liver cirrhosis, inflammation and other pathogenic factors activate hepatic stellate cells, increase collagen synthesis, and decrease collagen degradation. Collagen deposits in the space of Disse lead to the formation of basement membrane under endothelial cells in hepatic sinuses and a reduction in or disappearance of the upper fenestra, resulting in the formation of a diffuse barrier. This process is also known as hepatic sinusoidal capillarization. In addition, hepatic fibrosis leads to the formation of pseudolobules, which are typical histopathological features 
Table 6 Comparison of the four markers of hepatic fibrosis between groups $\left(\bar{x}_{ \pm \mathrm{S}}\right)$

\begin{tabular}{|c|c|c|c|c|}
\hline Indicators & $\mathrm{HA}(\mathrm{ng} / \mathrm{mL})$ & $\mathrm{LN}(\mathrm{ng} / \mathrm{mL})$ & PNIIINP (ng/mL) & CIV (ng/mL) \\
\hline Liver cirrhosis caused by hepatitis B & $165.29 \pm 74.39$ & $105.98 \pm 56.3$ & $147.74 \pm 83.02$ & $170.17 \pm 74.41$ \\
\hline Liver cirrhosis caused by hepatitis $C$ & $182.18 \pm 90.79$ & $83.64 \pm 27.81$ & $208.59 \pm 149.36$ & $187.44 \pm 45.34$ \\
\hline Alcoholic liver cirrhosis & $215.23 \pm 69.19$ & $132.29 \pm 62.41$ & $367.37 \pm 239.77$ & $289.01 \pm 203.29$ \\
\hline Autoimmune liver cirrhosis & $235.04 \pm 70.4$ & $240.67 \pm 172.27$ & $241.46 \pm 113.76$ & $286.39 \pm 179.54$ \\
\hline Occult liver cirrhosis & $139.25 \pm 81.39$ & $126.73 \pm 67.74$ & $143.7 \pm 73.36$ & $191.35 \pm 125.04$ \\
\hline Hepatoma caused by hepatitis B & $119.08 \pm 60.05$ & $94.61 \pm 49.59$ & $169.71 \pm 112.68$ & $147.83 \pm 88.4$ \\
\hline Hepatoma caused by hepatitis $C$ & $143.66 \pm 75.06$ & $144.83 \pm 92.12$ & $294.08 \pm 238.43$ & $278.55 \pm 247.18$ \\
\hline$P$ values & $<0.05$ & $<0.05$ & $<0.05$ & $<0.05$ \\
\hline
\end{tabular}

of liver cirrhosis (11).

Clinically, liver cirrhosis is diagnosed by the simultaneous presence of liver dysfunction and portal vein hypertension. According to the etiology, liver cirrhosis can be divided into viral liver cirrhosis, non-alcoholic liver cirrhosis, alcoholic liver cirrhosis, autoimmune liver cirrhosis, and congestive liver cirrhosis. In some patients, the development of liver cirrhosis cannot be explained by known etiologies; thus, it is termed occult liver cirrhosis (12). In China, viral hepatitis, of which hepatitis B is the most serious form, is the main cause of liver cirrhosis (13).

Liver biopsy is the gold standard for the diagnosis, classification, and staging of hepatic fibrosis; however, it is invasive and cannot be observed dynamically, and there are individual sampling differences (14). It has been confirmed that the concentrations of collagen, non-collagenous glycoproteins, and proteoglycans in the serum are significantly increased when hepatic fibrosis occurs. Serum HA, LN, PIIINP, and C IV have been used as serological markers to diagnose hepatic fibrosis, estimate the severity of the condition, and assess the prognosis for patients with chronic hepatic disease with better specificity and sensitivity (5).

Hyaluronic acid is synthesized by hepatic stellate cells and is an extracellular matrix component that can sensitively reflect the degree of fibrosis and damage to hepatocytes. Cai et al. (15) suggested that HA has a strong positive correlation with the degree of hepatic fibrosis compared with LN, PIIINP, and CIV. Laminin is a non- collagenous structural protein in the basement membrane, but it is expressed at a very low concentration in normal hepatic tissue. In hepatic fibrosis, $\mathrm{LN}$ deposits abnormally in hepatic sinuses, reduces the permeability of endothelial cells, and causes portal hypertension. Thus, it can reflect the degree of hepatic fibrosis (16).

Moreover, PIIINP can be used to achieve early diagnosis of hepatic fibrosis and evaluate the prognosis for patients with chronic hepatic disease. As a negative predictor of liver cirrhosis, it has a negative prediction rate of $>90 \%$. Therefore, PIIINP has certain application value in excluding diseases.

Synthesized by endothelial cells, which are the main component of basement membrane, the concentration of CIV might increase during proliferation of endothelial cells in the early stages of hepatic fibrosis and, together with LN, could promote capillarization of hepatic sinuses.

In the present study, the hospitalized patients were divided into groups according to their liver cirrhosis etiology. Serological hepatic function assays were carried out, and four markers of hepatic fibrosis were measured. The results showed that the hepatic function indices and four markers of hepatic fibrosis increased or decreased to varying degrees depending on the liver cirrhosis etiology.

The concentrations of ALT and AST were significantly different only in the liver cirrhosis caused by hepatitis B and hepatoma caused by hepatitis $\mathrm{B}$, with higher concentrations in the latter compared with the other groups. This finding indicated that if an increase in transaminase is observed in 
Table 7 The correlation analysis between the serum hepatic function indices and four hepatic fibrosis markers in the hepatoma caused by hepatitis B group

\begin{tabular}{|c|c|c|c|c|c|}
\hline Indicators & Values & HA & LN & PNIIIINP & CIV \\
\hline \multirow[t]{2}{*}{ ALT } & $r$ values & 0.132 & 0.104 & -0.151 & 0.031 \\
\hline & $P$ values & 0.430 & 0.533 & 0.365 & 0.853 \\
\hline \multirow[t]{2}{*}{ AST } & $r$ values & 0.428 & 0.458 & 0.219 & 0.374 \\
\hline & $P$ values & 0.007 & 0.004 & 0.187 & 0.021 \\
\hline \multirow[t]{2}{*}{ TBIL } & $r$ values & 0.228 & 0.480 & 0.38 & 0.415 \\
\hline & $P$ values & 0.168 & 0.002 & 0.017 & 0.010 \\
\hline \multirow[t]{2}{*}{ DBIL } & $r$ values & 0.266 & 0.457 & 0.406 & 0.400 \\
\hline & $P$ values & 0.107 & 0.004 & 0.011 & 0.013 \\
\hline \multirow[t]{2}{*}{ TP } & $r$ values & -0.270 & -0.103 & -0.213 & -0.234 \\
\hline & $P$ values & 0.101 & 0.540 & 0.200 & 0.157 \\
\hline \multirow[t]{2}{*}{ ALB } & $r$ values & -0.541 & -0.373 & -0.353 & -0.415 \\
\hline & $P$ values & 0.000 & 0.021 & 0.030 & 0.010 \\
\hline \multirow[t]{2}{*}{ PA } & $r$ values & -0.575 & -0.413 & -0.344 & -0.371 \\
\hline & $P$ values & 0.000 & 0.010 & 0.035 & 0.022 \\
\hline \multirow[t]{2}{*}{ ALP } & $r$ values & 0.233 & 0.235 & 0.033 & 0.071 \\
\hline & $P$ values & 0.158 & 0.155 & 0.845 & 0.673 \\
\hline \multirow[t]{2}{*}{ GGT } & $r$ values & -0.045 & 0.309 & 0.353 & 0.335 \\
\hline & $P$ values & 0.787 & 0.059 & 0.029 & 0.040 \\
\hline \multirow[t]{2}{*}{ TBA } & $r$ values & 0.218 & 0.283 & 0.363 & 0.419 \\
\hline & $P$ values & 0.188 & 0.085 & 0.025 & 0.009 \\
\hline \multirow[t]{2}{*}{ CHE } & $r$ values & -0.452 & -0.336 & -0.468 & -0.485 \\
\hline & $P$ values & 0.004 & 0.039 & 0.003 & 0.002 \\
\hline \multirow[t]{2}{*}{ AFU } & $r$ values & -0.050 & 0.072 & -0.216 & -0.110 \\
\hline & $P$ values & 0.766 & 0.668 & 0.192 & 0.512 \\
\hline \multirow[t]{2}{*}{ LAP } & $r$ values & 0.177 & 0.482 & 0.183 & 0.298 \\
\hline & $P$ values & 0.287 & 0.002 & 0.271 & 0.069 \\
\hline \multirow[t]{2}{*}{ SOD } & $r$ values & -0.334 & -0.347 & -0.487 & -0.536 \\
\hline & $P$ values & 0.040 & 0.033 & 0.002 & 0.001 \\
\hline
\end{tabular}

patients with hepatitis $\mathrm{B}$, this might indicate the progression of liver cirrhosis to hepatoma. Thus, dynamic detection of serological indicators and imaging are necessary to determine potential hepatic occupation.

The concentrations of TBIL, DBIL, and TBA increased in the liver cirrhosis and hepatoma with different etiologies. The most obvious increase occurred in the autoimmune liver cirrhosis group. Considering the patients in this group all had primary biliary liver cirrhosis, the three indices increased due to abnormal metabolism of bile acids. However, the sample size was small, and the sample type was single; thus, the present results can only be used as a reference.

Other markers to consider are ALP, GGT, ALB, PA, and 
Table 8 The correlation analysis between the serum hepatic function indices and four hepatic fibrosis markers in the hepatoma caused by hepatitis B group

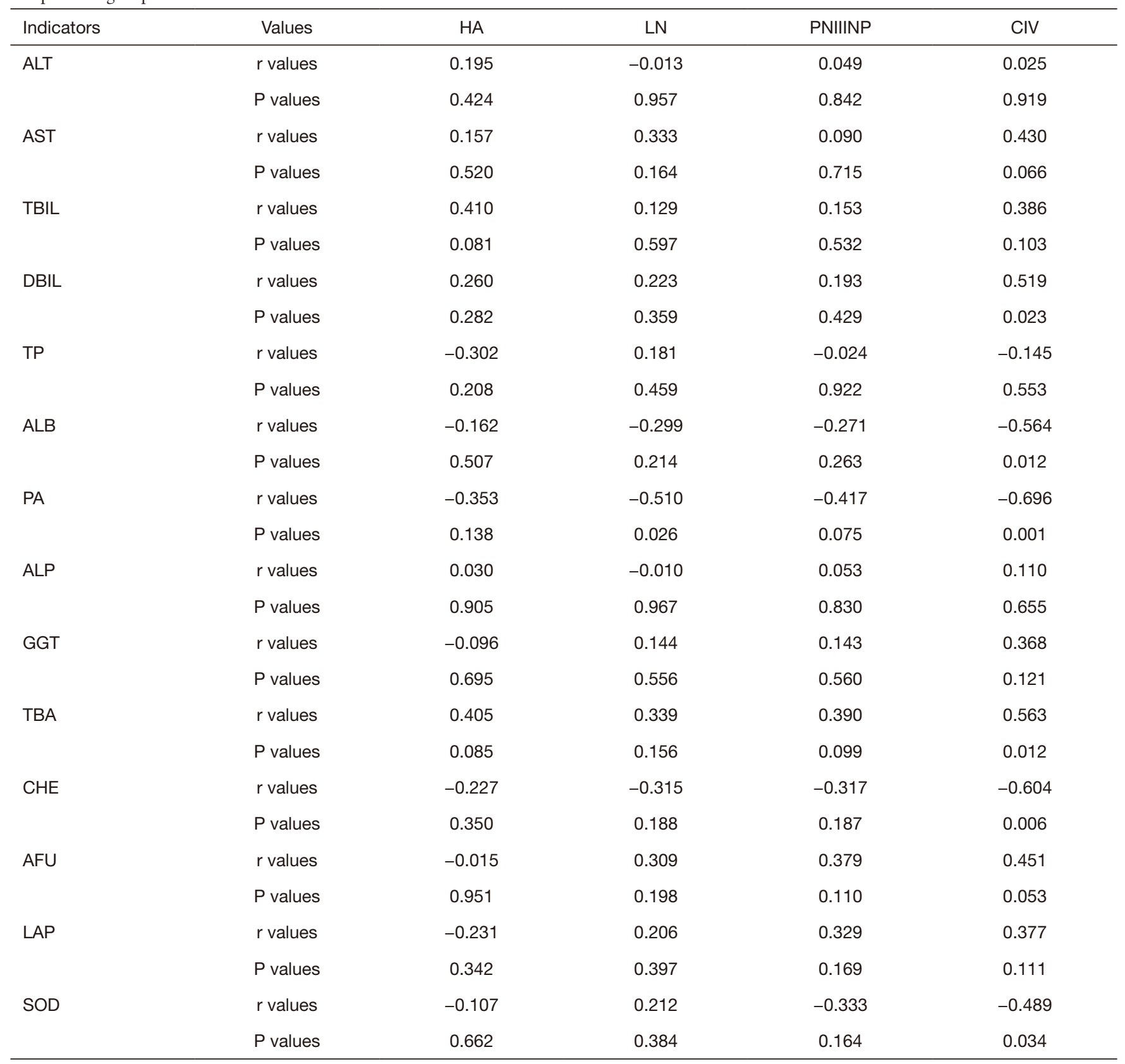

SOD. The concentration of SOD in each patient group was significantly lower compared with that in the control group. The latter observation indicated that the activity of antioxidant enzymes in hepatocytes is lower in injured hepatocytes compared with healthy hepatocytes. A decrease in SOD activity destroys the balance between free radical production and clearance and causes endothelial injury (17).
The concentration of $\mathrm{CHE}$ in the patients with liver cirrhosis was significantly lower in each patient group compared with the control group. Cholinesterase is synthesized by hepatocytes. During liver cirrhosis, with the necrosis of hepatocytes, infiltration of inflammatory cells, and proliferation of fibers and connective tissue, the synthesis of CHE might be affected, which could result 
in a decrease in its concentration (18). Moreover, the concentrations of the four markers of hepatic fibrosis were higher in each patient group compared with the control group.

Due to the small sample sizes of the alcoholic liver cirrhosis group, the group with cirrhosis caused by hepatitis $\mathrm{C}$, and the autoimmune liver cirrhosis group, the observations cannot be extrapolated to the whole population. Thus, only the correlation between the hepatic function indices and four hepatic fibrosis markers in the groups with liver cirrhosis caused by hepatitis B and hepatoma caused by hepatitis B were investigated. The results showed that AST was positively correlated with the four hepatic fibrosis markers in the former group, indicating that AST increases with an increase in hepatic injury together with an increase in the concentrations of $\mathrm{HA}, \mathrm{LN}$, PIIINP, and CIV.

There was a positive correlation between TBIL and DBIL and LN, PNIIINP, and CIV. This suggests that abnormal bile acid metabolism might accelerate collagen deposition and promote the progression of hepatic fibrosis. Moreover, TBA and GGT were positively correlated with PIIINP and CIV, while LAP was positively correlated with LN.

Furthermore, CHE, PA, ALB, and SOD were negatively correlated with hepatic fibrosis, indicating that necrosis and inflammatory infiltration of hepatocytes affect the synthesis or metabolism of these indices. With the aggravation of hepatic fibrosis, CHE, PA, ALB, and SOD decreased to varying degrees. However, there was no correlation between ALT, AST, TBIL, TP, ALP, and GGT and the four markers of hepatic fibrosis in the group with hepatoma caused by hepatitis B.

According to Zhu et al. (19), CHE can be used to predict significant hepatic fibrosis. In addition to CHE, the present study showed that PA, ALB, and SOD had a strong negative correlation with the four hepatic fibrosis markers in judging the degree of liver cirrhosis and hepatoma. The reason for this might be that PA, ALB, and CHE are indicators of protein synthesis and metabolism in hepatocytes. The decompensation of liver cirrhosis may lead to a decrease in hepatic functions (e.g., hepatic synthesis, bile metabolism), leading to an abnormal concentration of TBIL, TBA, ALB, and $\mathrm{PA}$, of which the former three increase while the latter two decrease.

Presently, several noninvasive serum markers are available to provide an assessment of liver fibrosis. The AST-to-platelet ratio index score, a publicly available formula consisting of the AST level and platelet count, could be used to evaluate liver fibrosis (20-22). The FIB4 index, consisting of age, AST, platelets, and ALT, is another publicly available formula to accurately identify individuals with severe fibrosis and cirrhosis $(23,24)$. The FibroTest formula includes macroglobulin, haptoglobin, apolipoprotein-A, glutamyl transferase, TBIL, age, and gender and can be used to risk-stratify liver disease ranging from mild disease to cirrhosis $(25,26)$. Study showed that noninvasive liver fibrosis assessment with FibroTest can reliably predict the five-year survival of patients with $\mathrm{HCV}$ infection. In further research, a combination of multiple biomarkers for liver fibrosis should be analyzed to improve the specificity.

There are differences between the results of the present study and those of other researchers, possibly due to differences in the sample sizes, selected indicators for research, and inclusion criteria. The present study comprised inpatients from our hospital only; thus, the sample does not represent the whole population.

\section{Conclusions}

In conclusion, in this study, the serological indices of liver cirrhosis of different etiologies significantly differed. Several serological indices, including the concentrations of ALT, AST, TBIL, DBIL, TBA, ALB, PA, CHE, GGT, and SOD, had a strong correlation with the four hepatic fibrosis markers (i.e., HA, LN, P III NP, C IV) during the progression of fibrosis. Therefore, combined detection could better reflect the progression of liver cirrhosis, improve the reliability and accuracy of liver cirrhosis diagnosis, guide clinical diagnosis and treatment, reduce the occurrence of hepatoma, and better judge the disease prognosis and curative effects of treatment.

\section{Acknowledgments}

We would like to acknowledge the hard and dedicated work of all the staff that implemented the intervention and evaluation components of the study.

Funding: None.

\section{Footnote}

Reporting Checklist: The authors have completed the MDAR checklist. Available at http://dx.doi.org/10.21037/ apm-20-1623 
Data Sharing Statement: Available at http://dx.doi. org/10.21037/apm-20-1623

Conflicts of Interest: All authors have completed the ICMJE uniform disclosure form (available at http://dx.doi. org/10.21037/apm-20-1623). The authors have no conflicts of interest to declare.

Ethical Statement: The authors are accountable for all aspects of the work in ensuring that questions related to the accuracy or integrity of any part of the work are appropriately investigated and resolved. The study was conducted in accordance with the Declaration of Helsinki (as revised in 2013). The study was approved by ethics board of Air Force Medical Center, PLA (approval number: 20161201) and informed consent was taken from all the patients.

Open Access Statement: This is an Open Access article distributed in accordance with the Creative Commons Attribution-NonCommercial-NoDerivs 4.0 International License (CC BY-NC-ND 4.0), which permits the noncommercial replication and distribution of the article with the strict proviso that no changes or edits are made and the original work is properly cited (including links to both the formal publication through the relevant DOI and the license). See: https://creativecommons.org/licenses/by-nc-nd/4.0/.

\section{References}

1. Bataller R, Brenner DA. Liver fibrosis. J Clin Invest 2005;115:209-18.

2. Tana MM, Muir AJ. Diagnosing Liver Fibrosis and Cirrhosis: Serum, Imaging, or Tissue? Clin Gastroenterol Hepatol 2018;16:16-8.

3. Tannapfel A, Dienes HP, Lohse AW. The indications for liver biopsy. Dtsch Arztebl Int 2012;109:477-83.

4. Yeom SK, Lee CH, Cha SH, et al. Prediction of liver cirrhosis, using diagnostic imaging tools. World J Hepatol 2015;7:2069-79.

5. Gao Q. Research Progress on Serologic Assays for Clinical Tests of Liver Fibrosis. Jilin Medicine 2011;6:1198-9.

6. Collazos J, Diaz F. Role of the measurement of serum procollagen type III Nterminal peptide in the evaluation of liver diseases. Clin Chim Acta 1994;227:37-43.

7. Lydatakis H, Hager IP, Kostadelou E, et al. Noninvasive markers to predict the liver fibrosis in non-alcoholic fatty liver disease. Liver Int 2006;26:864-71.
8. Kropf J, Gressner AM, Tittor W. Logistic-regression model for assessing portal hypertension by measuring hyaluronic acid (hyaluronan) and laminin in serum. Clin Chem 1991;37:30-5.

9. Wang WJ, Xiao P, Xu HQ, et al. Growing burden of alcoholic liver disease in China: A review. World J Gastroenterol 2019;25:1445-56.

10. Ahmed Z, Ahmed U, Walayat S, et al. Liver function tests in identifying patients with liver disease. Clin Exp Gastroenterol 2018;11:301-7.

11. Zhou WC, Zhang QB, Qiao L. Pathogenesis of liver cirrhosis. World J Gastroenterol 2014;20:7312-24.

12. Rinaldi L, Nascimbeni F, Giordano M, et al. Clinical features and natural history of cryptogenic cirrhosis compared to hepatitis $\mathrm{C}$ virus-related cirrhosis. World J Gastroenterol 2017;23:1458-68.

13. Chang B, Li B, Sun Y, et al. Changes in Etiologies of Hospitalized Patients with Liver Cirrhosis in Beijing 302 Hospital from 2002 to 2013. Mediators Inflamm 2017;2017:5605981.

14. Lo RC, Kim H. Histopathological evaluation of liver fibrosis and cirrhosis regression. Clin Mol Hepatol 2017;23:302-7.

15. Cai W, Tao J, Weng H. Influencing factor analysis of serum fibrosis indicators. Chinese Journal of Liver Disease 2003;1:23-5.

16. Lin Y. Research Progress on Serologic Assays for Clinical Tests of Liver Fibrosis. Chinese Medicine 2008;4:130-1.

17. Hu C, Luo J, Li J. Changes in serum SOD and VWF levels in patients with cirrhosis and their significance. Modern diagnosis and treatment 2015;11:2594-6.

18. Zhao F. Diagnostic value of CHE and ALB tests for cirrhosis (with 38 cases reported). Hubei Journal of Science and Technology (Medical Edition) 2006;3:255.

19. Zhu F, Zhu D, Chen J. Predictive value of commonly used serologic indicators on the extent of liver fibrosis. Journal of Radioimmunology 2013;4:392-5.

20. Maharaj B, Maharaj RJ, Leary WP, et al. Sampling variability and its influence on the diagnostic yield of percutaneous needle biopsy of the liver. Lancet 1986;1:523-5.

21. Wai CT, Greenson JK, Fontana RJ, et al. A simple noninvasive index can predict both significant fibrosis and cirrhosis in patients with chronic hepatitis C. Hepatology 2003;38:518-26.

22. Bonder A, Tapper EB, Afdhal NH. Contemporary assessment of hepatic fibrosis. Clin Liver Dis 2015;19:123-34. 
23. Vallet-Pichard A, Mallet V, Nalpas B, et al. FIB-4: an inexpensive and accurate marker of fibrosis in $\mathrm{HCV}$ infection: comparison with liver biopsy and fibrotest. Hepatology 2007;46:32-6.

24. Sterling RK, Lissen E, Clumeck N, et al. Development of a simple noninvasive index to predict significant fibrosis in patients with $\mathrm{HIV} / \mathrm{HCV}$ coinfection. Hepatology
2006;43:1317-25.

25. Poynard T, Morra R, Halfon P, et al. Meta-analyses of FibroTest diagnostic value in chronic liver disease. BMC Gastroenterol 2007;7:40.

26. Imbert-Bismut F, Ratziu V, Pieroni L, et al. Biochemical markers of liver fibrosis in patients with hepatitis $\mathrm{C}$ virus infection: a prospective study. Lancet 2001;357:1069-75.

Cite this article as: Ma HY, Dong L, Quan SZ, Li RY, Wang XR. Comparison of four markers of hepatic fibrosis and hepatic function indices in patients with liver cirrhosis and hepatoma. Ann Palliat Med 2021;10(4):4108-4121. doi: 10.21037/apm-20-1623 